\title{
Effect of Medication Copayment on Adherence and Discontinuation in Medicare Beneficiaries with Type 2 Diabetes: A Retrospective Administrative Claims Database Analysis
}

\author{
Manjiri D. Pawaskar · Liou Xu • Yuexin Tang - Gary A. Puckrein • \\ Swapnil N. Rajpathak · Bruce Stuart
}

Received: June 28, 2018 / Published online: August 24, 2018

(C) The Author(s) 2018

\section{ABSTRACT}

Introduction: Nonadherence to antihyperglycemic agents (AHAs) increases the incidence of morbidity and mortality, as well as healthcare-related costs, in patients with type 2 diabetes (T2D). This study examined the association between medication copayment and adherence and discontinuation among elderly patients with T2D who use generic versus branded AHAs.

Methods: A retrospective, observational cohort study used Medicare administrative claims data (index period: 1 June 2012 to 31 December 2013). Drug copayments were measured as the copayment of the index medication for a 30-day supply after patients met their plan deductible.

Enhanced Digital Features To view enhanced digital features for this article go to https://doi.org/10.6084/ m9.figshare.6940760.

Electronic supplementary material The online version of this article (https://doi.org/10.1007/s13300$018-0489-y)$ contains supplementary material, which is available to authorized users.

M. D. Pawaskar $(\bowtie) \cdot$ Y. Tang · S. N. Rajpathak

Merck \& Co., Inc., Kenilworth, NJ, USA

e-mail: Manjiri.pawaskar@merck.com

L. Xu · G. A. Puckrein

National Minority Quality Forums, Washington,

DC, USA

B. Stuart

University of Maryland, Baltimore, MD, USA
Patients were stratified into a branded or generic cohort based on the index medication. Adherence was measured by the proportion of days covered $(\geq 80 \%)$ and discontinuation by a treatment gap of $>60$ days in 10 months during the follow-up period. Poisson regressions were conducted for medication adherence and discontinuation, while controlling for demographic, clinical, and comorbid conditions.

Results: Overall, 160,250 patients on AHA monotherapy were included in the analysis; $131,594(82 \%)$ were prescribed a generic and $28,656(18 \%)$ a branded AHA with a mean copay of $\$ 6$ and $\$ 41$, respectively. Increases in copayment increased nonadherence and discontinuation for branded medications but not for generic AHA medications. In both cohorts, elderly patients ( $\geq 75$ years of age) had a lower risk of nonadherence and discontinuation. Black patients had a higher risk of nonadherence or discontinuing medication. Patients having more frequent inpatient, emergency room, and/or physician visits were at higher risk of nonadherence or discontinuing therapy in the branded and generic cohorts $(P<0.001)$.

Conclusion: The impact of drug copayment on adherence and discontinuation varied considerably between branded and generic AHAs. Medicare patients taking branded AHAs had a higher risk of nonadherence with increasing copayment and were more likely to discontinue medication, whereas this association was not observed in patients taking generic medications. 
Funding: Merck \& Co, Inc., Kenilworth, NJ, USA.

Plain Language Summary: Plain language summary available for this article.

Keywords: Adherence; Copayment; Medicare; Type 2 diabetes

\section{PLAIN LANGUAGE SUMMARY}

People with type 2 diabetes take medicine to control their blood sugar to avoid poor health results. This study investigated whether the price the patient paid for their pills was linked to whether they took their pills as their doctor ordered or stopped taking them. The results showed that people who had to pay more money for their brand name pills (mean, \$41) did not take them as their doctor ordered or stopped taking them. This did not tend to be true if the pills cost $\$ 10$ or less. Going to the hospital, emergency room, and the doctor's office often were also linked to not following doctor's orders or stopping their pills. The data from this study are key for payers to bear in mind when making decisions.

\section{INTRODUCTION}

The Centers for Disease Control and Prevention estimates that 30.3 million people in the United States (9.4\%) had diabetes in 2015 [1]. The proportion of patients with diabetes increases with age; 12 million patients aged $\geq 65$ years have diabetes (i.e., $25 \%$ of the elderly population) [1]. Most have type 2 diabetes (T2D), which is the most prevalent form, representing $90-95 \%$ of all patients with diabetes [1].

Uncontrolled, elevated blood glucose is associated with increased complications including myocardial infarction, stroke, microvascular disease, and mortality [2]. Adherence to antihyperglycemic agents (AHAs) helps maintain glycemic control $[3,4]$ and prevents or minimizes T2D-related complications [2]. Conversely, nonadherence has been shown to substantially increase the incidence of morbidity and mortality as well as healthcare costs $[2,5]$.
Data from the National Health and Nutrition Examination Survey show that only approximately half of patients with T2D achieve the recommended glycated hemoglobin (HbA1c) target of $<7.0 \%$ [6], and the Healthcare Effectiveness Data and Information Set (HEDIS) database shows that only approximately $32 \%$ of government-insured patients and $37 \%$ of commercially insured patients achieve this HbA1c goal [7]. The enormity of the problem of obtaining the target HbA1c may be even greater than shown in the published literature. A recent retrospective cohort study reported that despite the findings of many randomized controlled trials of new AHAs showing significant HbA1c reductions, improvements in clinical outcomes in the real-world setting have not mirrored those observed in the study setting. The primary cause for this discrepancy appears to be poor medication adherence [8].

A validated health economics diabetes model [IMS CORE Diabetes Model (IMS Disease Analyzer-UK database)] [9] demonstrated that readily achievable improvements in glycemic control would result in significant reductions in the incidence of microvascular complications, which should decrease costs related to diabetes (types 1 and 2) [10]. Because increased adherence decreases diabetes-associated complications, both increased cost savings and improved clinical outcomes would be expected to follow improved glycemic control. Curtis et al. investigated the relationship between adherence to AHAs and outcomes in patients with T2D $(N=228,074)$ in a retrospective analysis of a US claims database (Truven Health Analytics MarketScan ${ }^{\circledR}$ Commercial Claims and Encounters database; included data from 1 July 2009, through 30 June 2014) [11]. Analysis of patient medical records showed that adherence resulted in statistically significantly lower acute care and outpatient costs; lower probability of, and shorter length of, hospital stays; and fewer hospitalizations (all $P<0.0001$ ) compared with nonadherent patients [11].

Numerous factors can negatively impact adherence, including prescription drug copayments. A retrospective cohort analysis showed that medication adherence decreased in patients with diabetes who were receiving AHAs 
through a Medicare Part D coverage plan after entering the coverage gap (i.e., "donut hole") wherein copayments increased, particularly for those with coverage for only generic medications [12]. A retrospective, observational analysis of medical and pharmacy claims data from Pittsburgh Plate Glass (PPG) Industries employees, retirees, and dependents (2003-2005) that stratified copayments by monthly cost showed that high copayments were associated with lower adherence to oral diabetes medications irrespective of age [13]. An additional retrospective analysis of patients from seven commercial health plans in the United States who had been newly initiated on an oral AHA reported that in patients with $\mathrm{T} 2 \mathrm{D}$ who had received $\geq 1$ oral AHA, an increase in the copayment for their medication was a statistically significant $(P<0.0001)$ predictor of treatment failure and corresponded with a higher proportion of patients discontinuing therapy for each higher copayment category [14].

Limited data are available on the effect of medication copayment on adherence and discontinuation in elderly or disabled patients, particularly those with diabetes, as well as how this association may be impacted by the use of generic versus branded medications. The purpose of this retrospective database analysis was to examine the association between medication copayment and adherence and discontinuation among Medicare beneficiaries with T2D who use (1) generic and (2) branded AHAs.

\section{METHODS}

\section{Study Design}

\section{Data Source}

The primary data source for this retrospective, observational cohort study was administrative claims data from Medicare Part A, B, and D Event Files and the Medicare 100\% Master Beneficiary Summary File Chronic Condition Segment file from 1 June 2012 to 31 December 2013. Individual level claims data were obtained from the Chronic Condition Data Warehouse RIF files. Both the Chronic Condition Warehouse and Part D event files contain a record for every prescription drug claim or doctor's visit. The Master Beneficiary Summary File served as an intermediate set of files that linked all the records together by their common unique beneficiary identification numbers. The master beneficiary summary file also included variables such as date of birth, gender, race/ethnicity, and zip code of residence, which allowed us to identify the demographic and geographic information of the T2D cases. These data cover 39 million Medicare beneficiaries in the United States and represent $100 \%$ of Medicare fee-forservice beneficiaries, including 8 million beneficiaries with T2D. Although the Medicare data used in this analysis included beneficiaries' demographic, clinical, and health characteristics, there was no access to individual patient records. The Medicare data are maintained by the National Minority Quality Forum under a data use agreement with the Center for Medicare and Medicaid Services. The data were cleaned and checked for consistency in the course of data preparing. Inconsistencies and missing values that appeared during the study period were removed during the cleaning process.

The primary independent variable in this study was drug copayments for the index AHA under Medicare Part D. The copayment was measured as the copayment of index medication for a 30-day supply after patients had met their deductible amount in the plan. Patients who did not meet the deductible during the study period had a missing copayment value and thus were not included in the analysis.

The index period comprised the first 2 months of the calendar year (i.e., January 1-February 28, 2013) and was considered adequate to obtain a sufficient number of patients for the study after implementation of the approved new formulary structure (implemented 1 January) for that same year. Patients were then monitored for 10 months after the index date in the same calendar year to avoid the influence of any changes in health plans in a different enrollment year.

Patients were stratified into either a branded or generic cohort based on their index medication. Patient-level characteristics at baseline were measured within 6 months before the 
index date, which included both demographics and comorbid conditions. Patients were subsequently monitored for 10 months after the index date to capture their adherence and discontinuation rates.

\section{Population}

The study population was composed of Medicare fee-for-service beneficiaries aged 18 years or older with a diagnosis of T2D [International Classification of Diseases, Ninth Revision (ICD9) code 250.x0 or 250.x2] at baseline who had at least one prescription for non-insulin AHA monotherapy under Medicare Part D coverage during the 16-month study period. All patients had continuous coverage under the Medicare Part A, B, and D plan and were required to take the same AHA medication during the follow-up period as in the index period (i.e., remaining on monotherapy). Exclusion criteria included a diagnosis of type 1, gestational, or secondary diabetes; patients on insulin therapy during the index period; Medicare Advantage recipients; low-income subsidiary recipients; patients who died during the 16-month study period; patients who had a missing value for a copayment; and patients with extreme copayment values of the 99th percentile or above. This article is based on administrative claims data and does not contain any studies with human participants performed by any of the authors.

\section{Outcomes}

Adherence to an index AHA was estimated as the proportion of days covered (PDC), which was calculated as the total number of days covered by medication, divided by the number of days in the follow-up period [15]. Adherence to the index AHA was defined as PDC $\geq 80 \%$. Patients switching to another agent within the same drug class during the study were still considered adherent as long as the PDC was $\geq 80 \%$. Nonadherence to the index AHA was defined as PDC $<80 \%$. PDC values could range from $0 \%$ (poor adherence) to 100\% (complete adherence). Adherence to the index AHA was assessed as a binary variable. Discontinuation, or non-persistency, from the index AHA was measured as a binary variable and estimated as a treatment gap of $>60$ days [15].

\section{Covariates}

Key demographic and clinical variables included age, sex, race/ethnicity, and geographic region. Disease severity and overall health status were adjusted for by including ICD-9 codes for comorbidities and diabetes complications as a measure of disease severity. Comorbidities were also evaluated using the Charlson Comorbidity Index (the greater the total Charlson Index score, the greater the comorbidity burden on the patient) [16]. Prescription drug plan information included the Part D plan information of the patient and Part D benefit types, such as enhanced alternative versus all others and no benefit plan information versus all others. Covariate indicated whether the patient was a new or prevalent user of the index AHA. Other covariates were used to cover the clinical and health status during the preindex period, including the total number of medications used (diabetes and non-diabetes), number of hospitalizations, emergency room (ER) visits and physician events, and the total out-ofpocket Part D costs.

\section{Statistical Analysis}

Analyses included descriptive statistics (mean \pm standard deviation) for all measured outcomes. To assess the primary objective of determining the effects of copayments on outcomes, copayment amounts were examined using copayments as a categorical variable; different cutpoints were applied to the branded cohort [ $\$ 0-\$ 10$ (reference level); \$10.01-\$20; \$20.01-\$30; \$30.01-\$40; \$40.01-\$50; \$50.01$\$ 60$; and $>\$ 60$ ] and generic cohort [ $\$ 0$ (reference level); \$0.01-\$2; \$2.01-\$4; \$4.01-\$6; $\$ 6.01-\$ 8 ; \quad \$ 8.01-\$ 10 ;>\$ 10]$. The effects of copayments on outcomes were also examined by applying the same cutpoints to the branded and generic cohort [ $\$ 0-\$ 5$ (reference level); \$5.01-\$10; \$10.01-\$15; \$15.01-\$20; \$20.01$\$ 25 ; \quad \$ 25.01-\$ 30 ; \quad \$ 30.01-\$ 35 ; \quad \$ 35.01-\$ 40 ;$ $\$ 40.01-\$ 45 ; \$ 45+]$. An additional regression 
analysis was performed that used copayment as a continuous variable to assess the effect of copayment on medication adherence and discontinuation of medication. Bivariate analysis was performed to assess cohort differences in all relevant demographic and clinical characteristics as well as adherence and discontinuation rates. Cohort differences in these variables were assessed via chi-square tests and $t$ tests or analysis of variance for categorical and continuous variables, respectively.

To examine the relationship between copayment and outcomes (e.g., adherence and discontinuations), Poisson regression was performed, controlling for patient baseline characteristics reported under covariates.

\section{RESULTS}

Overall, 795,848 elderly beneficiaries enrolled for 2012 were adults with T2D who had at least one prescription for an AHA therapy during the index period. After applying exclusion criteria to this population, 160,250 patients were on AHA monotherapy and met the criteria for inclusion in the analysis. Of these patients, $131,594(82 \%)$ were prescribed a generic AHA, and $28,656(18 \%)$ were prescribed a branded AHA.

Demographic and baseline characteristics for this subpopulation are shown in Table 1. Patient characteristics were similar between both the generic and branded cohorts. Patients aged $\geq 75$ years represented approximately $62 \%$ of the study population in the generic cohort compared with $65 \%$ in the branded cohort. There were more women than men in both the generic $(52.1 \%$ vs. $47.9 \%$, respectively) and branded (53.1\% vs. $46.9 \%)$ cohorts (Table 1$)$.

Overall, patients in the generic cohort had better health status versus the branded cohort. Comparing the generic with the branded cohort, the generic cohort had fewer comorbidities, fewer diabetes-related complications $[\geq 2$ complications, $n=6929 \quad(5.3 \%) \quad$ vs. $n=2163$ (7.5\%), respectively], and a lower Charlson Index score (1.02 vs. 1.31) and tended to consume fewer healthcare resources [i.e., mean (SD) hospital visits, $0.24(0.5)$ vs 0.27
(0.6), $P<0.0001$; ER visits, 0.38 (0.7) vs. 0.42 (0.7), $P<0.0001$; and physician visits, 5.24 (3.9) vs. 6.26 (4.4), $P<0.0001]$ as well as number of prescription drugs used [8.9 (4.0) vs. 9.5 (4.3), $P<0.0001$; Table 1]. In addition, a greater proportion of patients had chronic kidney disease in the branded versus generic cohort (34.9\% vs, 23.3\%, respectively; Table 1 ).

The mean total per-patient out-of-pocket Part D prescription cost during the preindex period for the study population was $\$ 517.39$, with the total cost in the branded cohort almost double the cost in the generic cohort ( $\$ 861.54$ vs. $\$ 442.45$, respectively; Table 1 ). The branded drug cohort copayment ranged from $\$ 0$ to $\$ 106.57$ with a mean copayment value of $\$ 40.79$ (Supplemental Figure S1A). In comparison, the drug copayment for the generic cohort had a skewed distribution with a long right tail, ranging from $\$ 0$ to $\$ 106.15 ; 90 \%$ of the values were below $\$ 10.00$ (Supplemental Figure S1B).

An analysis of the association between medication copayment and adherence and discontinuation rates among Medicare beneficiaries with T2D who use generic versus branded AHA monotherapy using the copayment cutpoints in Fig. 1 showed that although an increase in drug copayment was associated with lower drug adherence and a higher drug discontinuation rate for elderly beneficiaries on branded AHA monotherapy, this same pattern was not observed for beneficiaries on generic AHA monotherapy. Moreover, relative risk increased with a higher copayment in patients on branded monotherapy, whereas no consistent pattern was seen in the generic cohort. For example, in the highest copayment subgroup (> \$60) for patients on branded medication, patients were 2.1 times more likely to be nonadherent with their medication (Fig. 1b) and 1.9 times more likely to discontinue (Fig. 1b) a branded AHA compared with patients having a copayment of $\leq \$ 10$.

Among the modeled covariates investigated in this study, age, sex, race/ethnicity, region, and health resource utilization in the preindex period were shown to be statistically significant predictors for AHA nonadherence (Figs. 2, 3). Elderly patients (aged $\geq 75$ years) had a lower probability of being nonadherent or 
Table 1 Demographics, economic characteristics, and baseline clinical characteristics

\begin{tabular}{|c|c|c|c|c|}
\hline Variable & $\begin{array}{l}\text { Overall cohort } \\
(N=160,250)\end{array}$ & $\begin{array}{l}\text { Branded cohort } \\
(n=28,656)\end{array}$ & $\begin{array}{l}\text { Generic cohort } \\
(n=131,594)\end{array}$ & $P$ value \\
\hline \multicolumn{5}{|l|}{ Demographics and baseline characteristics } \\
\hline Age, years, mean $(\mathrm{SD})$ & $77.3(7.2)$ & $77.9(6.9)$ & $77.2(7.2)$ & $<0.0001^{\mathrm{a}}$ \\
\hline \multicolumn{5}{|l|}{ Sex, $n(\%)$} \\
\hline Men & $76,419(47.7)$ & $13,446(46.9)$ & $62,973(47.9)$ & $0.0042^{\mathrm{a}}$ \\
\hline Women & $83,831(52.3)$ & $15,210(53.1)$ & $68,621(52.1)$ & \\
\hline \multicolumn{5}{|l|}{ Race/ethnicity, $n$ (\%) } \\
\hline White & $144,178(90.0)$ & $25,837(90.2)$ & $118,341(89.9)$ & $0.0007^{\mathrm{a}}$ \\
\hline Black & $10,210(6.4)$ & $1707(6.0)$ & $8503(6.5)$ & \\
\hline Asian & $1543(1.0)$ & $302(1.1)$ & $1241(0.9)$ & \\
\hline Hispanic & $1002(0.6)$ & $173(0.6)$ & $829(0.6)$ & \\
\hline Other & $3223(2.0)$ & $611(2.1)$ & $2612(2.0)$ & \\
\hline Unknown & $94(0.1)$ & $26(0.1)$ & $68(0.1)$ & \\
\hline \multicolumn{5}{|l|}{ Geographic region, $n(\%)$} \\
\hline Northeast & $30,146(18.8)$ & $7591(26.5)$ & $22,555(17.1)$ & $<0.0001^{\mathrm{a}}$ \\
\hline Midwest & $41,501(25.9)$ & $6032(21.0)$ & $35,469(27.0)$ & \\
\hline South & $66,321(41.4)$ & $11,337(39.6)$ & $54,984(41.8)$ & \\
\hline West & $22,282(13.9)$ & $3696(12.9)$ & $18,586(14.1)$ & \\
\hline New index AHA user, $n(\%)$ & $20,533(12.8)$ & $3511(12.3)$ & $17,022(12.9)$ & $0.0017^{\mathrm{a}}$ \\
\hline $\begin{array}{l}\text { Number of medications used in preindex } \\
\text { period, mean }(\mathrm{SD})\end{array}$ & $9.01(4.02)$ & $9.53(4.31)$ & $8.90(3.95)$ & $<0.0001^{\mathrm{a}}$ \\
\hline \multicolumn{5}{|c|}{ Healthcare resource utilization preindex, mean (SD) } \\
\hline Hospitalizations & $0.24(0.52)$ & $0.27(0.55)$ & $0.24(0.51)$ & $<0.0001^{\mathrm{a}}$ \\
\hline Emergency room visits & $0.39(0.67)$ & $0.42(0.70)$ & $0.38(0.67)$ & $<0.0001^{\mathrm{a}}$ \\
\hline Physician visits & $5.42(4.00)$ & $6.26(4.43)$ & $5.24(3.88)$ & $<0.0001^{\mathrm{a}}$ \\
\hline $\begin{array}{l}\text { Total out-of-pocket cost preindex } \\
\text { (medical }+ \text { drug), \$, mean (SD) }\end{array}$ & $1369.62(1345.23)$ & $1814.37(1434.73)$ & $1272.77(1305.00)$ & $<0.0001^{\mathrm{a}}$ \\
\hline $\begin{array}{l}\text { Total out-of-pocket Part D prescription } \\
\text { cost preindex, \$, mean }(\mathrm{SD})\end{array}$ & $517.39(470.12)$ & $861.54(507.91)$ & $442.45(426.10)$ & $<0.0001^{\mathrm{a}}$ \\
\hline
\end{tabular}


Table 1 continued

\begin{tabular}{|c|c|c|c|c|}
\hline Variable & $\begin{array}{l}\text { Overall cohort } \\
(N=160,250)\end{array}$ & $\begin{array}{l}\text { Branded cohort } \\
(n=28,656)\end{array}$ & $\begin{array}{l}\text { Generic cohort } \\
(n=131,594)\end{array}$ & $P$ value \\
\hline \multicolumn{5}{|l|}{ Clinical characteristics-comorbidities } \\
\hline Charlson Comorbidity Index, mean (SD) & $1.07(1.48)$ & $1.31(1.62)$ & $1.02(1.44)$ & $<0.0001^{\mathrm{a}}$ \\
\hline \multicolumn{5}{|l|}{ Comorbidity, $n$ (\%) } \\
\hline Cardiovascular disease & $59,838(37.3)$ & $11,869(41.4)$ & $47,969(36.5)$ & $<0.0001^{\mathrm{a}}$ \\
\hline Retinopathy & $11,453(7.1)$ & $2302(8.0)$ & $9151(7.0)$ & $<0.0001^{\mathrm{a}}$ \\
\hline Neuropathy & $26,088(16.3)$ & $5059(17.7)$ & $21,029(16.0)$ & $<0.0001^{\mathrm{a}}$ \\
\hline Chronic kidney disease & $40,646(25.4)$ & $9995(34.9)$ & $30,651(23.3)$ & $<0.0001^{\mathrm{a}}$ \\
\hline Hypertension & $136,565(85.2)$ & $25,054(87.4)$ & $111,511(84.7)$ & $<0.0001^{\mathrm{a}}$ \\
\hline Hyperlipidemia & $121,330(75.7)$ & $23,127(80.7)$ & $98,203(74.6)$ & $<0.0001^{\mathrm{a}}$ \\
\hline Obesity/overweight & $8795(5.5)$ & $1688(5.9)$ & $7107(5.4)$ & $0.0010^{\mathrm{a}}$ \\
\hline Depression & $18,618(11.6)$ & 3413 (11.9) & $15,205(11.6)$ & 0.0885 \\
\hline $\begin{array}{l}\text { Number of diabetes-related complications, } \\
\text { mean (SD) }\end{array}$ & $0.36(0.62)$ & $0.43(0.68)$ & $0.34(0.61)$ & $<0.0001^{\mathrm{a}}$ \\
\hline
\end{tabular}

$A H A$ antihyperglycemic agent, $S D$ standard deviation

a Statistically significant at the 0.01 level

discontinuing the index AHA irrespective of whether it was a generic or branded AHA (Figs. 2, 3). Black patients had a higher risk of being nonadherent or discontinuing their medication compared with white patients for both generic and branded AHAs (Figs. 2, 3) whereas Hispanic patients had a higher risk of nonadherence and discontinuing generic AHAs compared with white patients (Fig. 3). Male patients had a lower risk for nonadherence and medication discontinuation only for generic AHAs (Fig. 3). In addition, patients who newly initiated treatment with an index AHA were more likely to be nonadherent or discontinue AHA therapy. In addition, patients who had previous hospitalizations and/or physician visits were at a statistically significantly $(P<0.001)$ higher risk of being nonadherent or discontinuing therapy in both the branded and generic cohorts (Figs. 2, 3).

An analysis of the association between medication copayment and adherence and discontinuation rates using the same copayment cutpoints for both generic and branded AHA monotherapy is shown in Supplemental Tables S1 and S2. A greater trend toward higher copayments increasing the relative risk of being nonadherent was observed in the branded cohort compared with the generic cohort with relative risk being greater in the branded cohort for every copayment cutpoint greater than $\$ 15$. For the highest copayment group, patients in the branded AHA monotherapy group were 1.8 times more likely to be nonadherent with their medication versus 1.2 times in the generic cohort. A similar trend was observed for discontinuation rates (Supplemental Tables S1 and S2). As observed in the previous analysis, age, sex, race/ethnicity, region, and health resource utilization in the preindex period were shown to be statistically significant predictors for AHA nonadherence among the modeled covariates investigated in this study (Supplemental Tables S1 and S2). Similar to the above analyses, results of a regression analysis that used copayment as a continuous variable showed that the relative risk for being nonadherent or discontinuing medication decreased with increasing 


\section{A Generic cohort $(n=131,594)$}

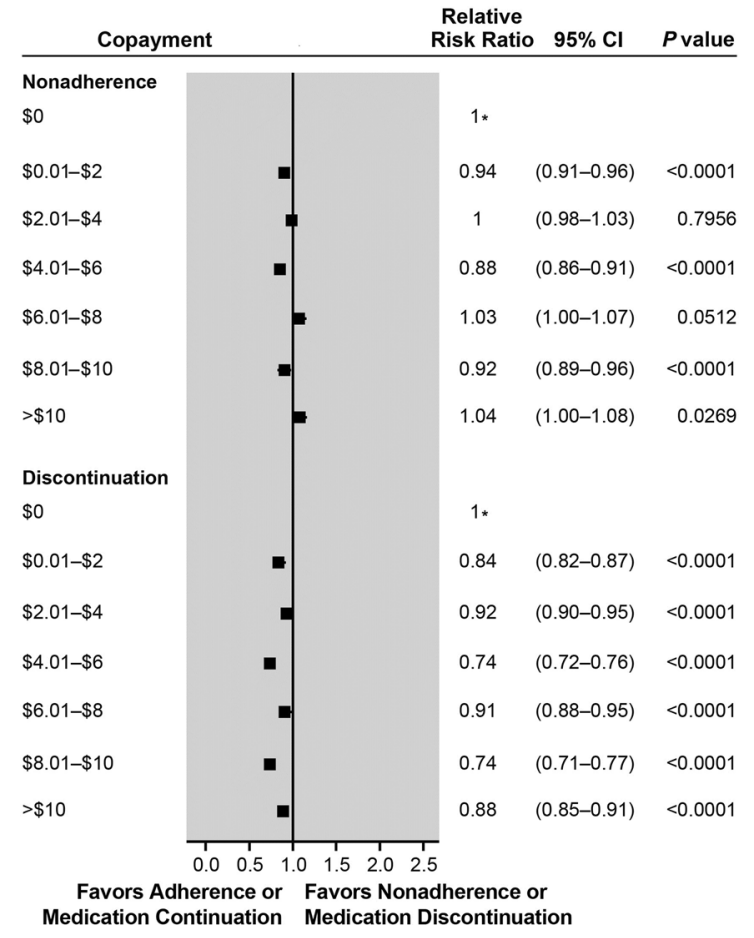

Fig. 1 Relative risk for AHA medication nonadherence and discontinuation in the a generic medication cohort and $\mathbf{b}$ branded medication cohort for patients with type 2 diabetes based on Medicare copayment. AHA antihyperglycemic agent, $C I$ confidence interval. Asterisk: reference category. The following covariates were controlled in the

age in both the generic and branded cohorts (Supplemental Tables S3 and S4).

Calculation of the overall impact for the raw linear effect of copayment on medication adherence and discontinuation showed that in the branded cohort the risk of nonadherence would increase by 0.9 percent (relative risk = 1.009) with every dollar increase in copayment whereas the risk of discontinuation would increase by 0.8 percent (relative risk $=1.008$ ) with every dollar increase (Supplemental Tables S3 and S4).

\section{DISCUSSION}

Limited published data are available on the effect of medication copayment on adherence and discontinuation in Medicare beneficiaries
B Branded cohort $(n=28,656)$

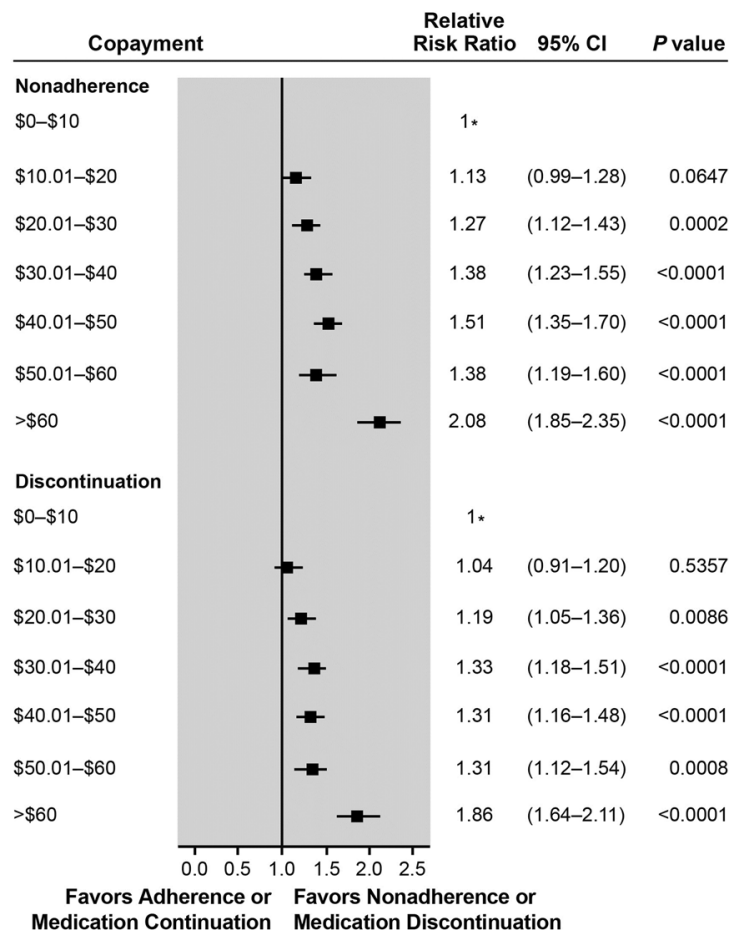

model at baseline: demographics (age, gender, race, and geographic regions), clinical factors (comorbidities, Charlson Comorbidity Index, and diabetes-related complications), and economic factors (healthcare resource utilization, medication utilization, new AHA users, Part $\mathrm{D}$ benefit plan type, and out-of-pocket Part D costs)

with diabetes. We believe the current analysis is the first study to utilize a large beneficiary database comprised of a 100\% Medicare fee-forservice population to investigate the effect of medication usage (branded vs generic) and copayment amount on AHA adherence and discontinuation as well as the potential economic burden that copayments place on beneficiaries with T2D. This aspect of the costsharing strategy was evaluated using the Medicare Part D plan. Data from this study on index AHA monotherapies in patients with T2D suggest that the impact of drug copayment on adherence and discontinuation varies considerably between branded and generic AHAs among Medicare patients. Medicare patients taking branded AHAs had a higher risk of being nonadherent with increasing copayment as well as being more likely to discontinue medication. 


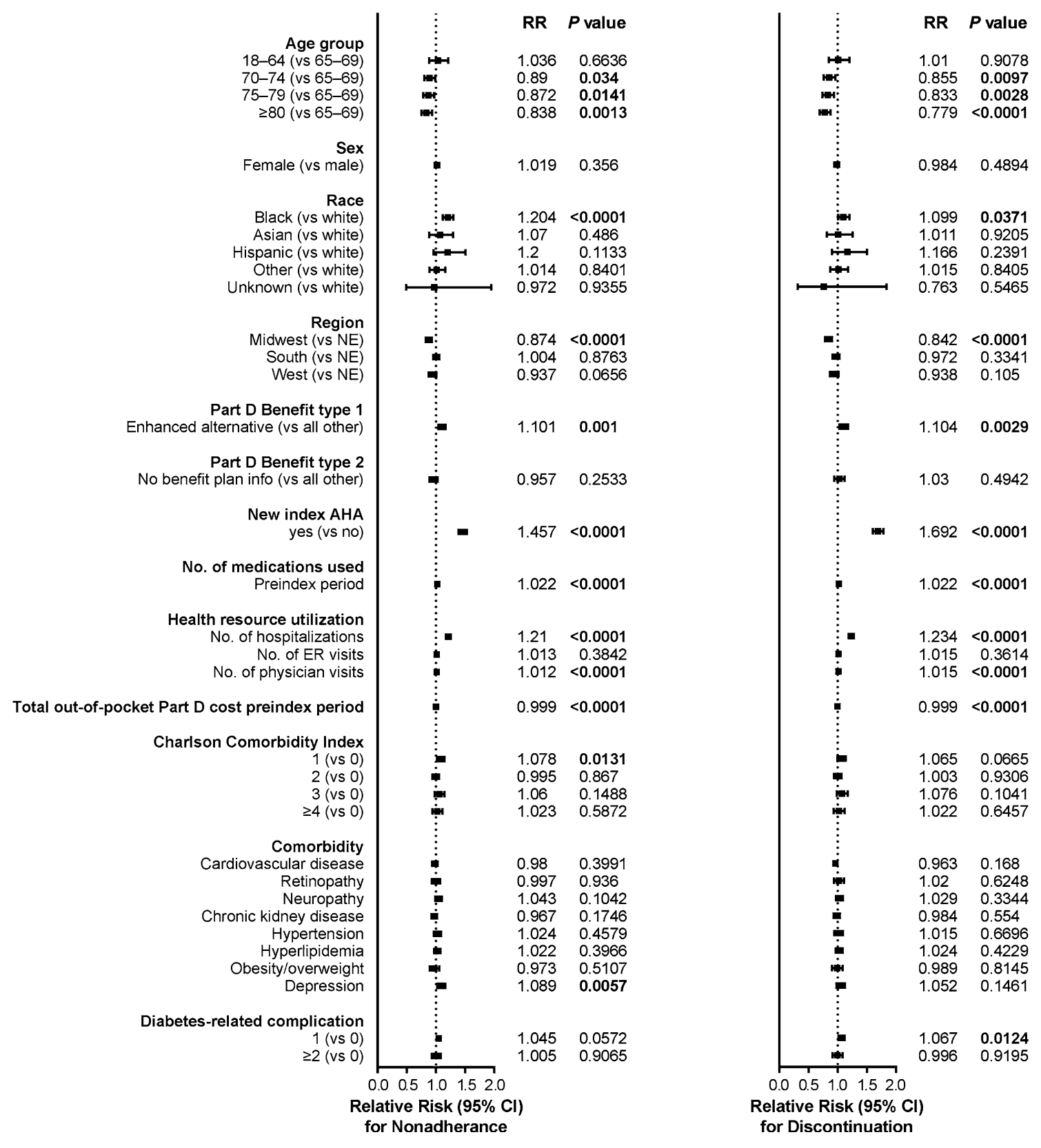

Fig. 2 Relative risk for nonadherence and discontinuation for the branded medication cohort. AHA antihyperglycemic agent, $C I$ confidence interval, $E R$ emergency room, $N E$ Northeast, $R R$ relative risk. The following covariates were controlled in the model at baseline: demographics (age, gender, race, and geographic regions),

clinical factors (comorbidities, Charlson Comorbidity Index, and diabetes-related complications), and economic factors (healthcare resource utilization, medication utilization, new AHA users, Part D benefit plan type, and out-ofpocket Part D costs)

Patients taking generic medications did not show a similar pattern of nonadherence or discontinuation. This observed pattern was similar irrespective of whether the same or different

copayment categories were used for the branded and generic cohort regression analyses. A possible explanation for this finding could be that the mean copayment cost for branded 


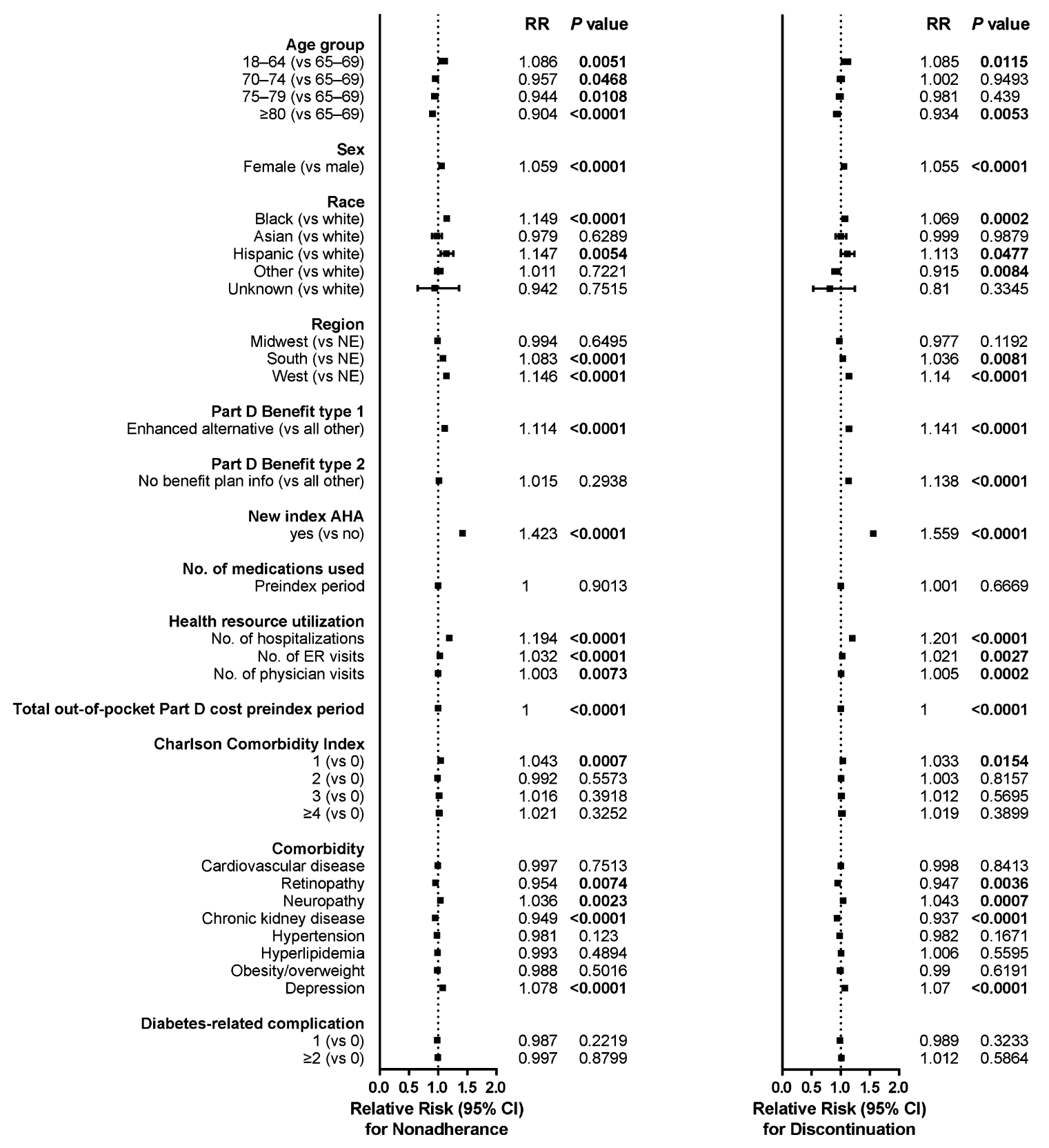

Fig. 3 Relative risk for nonadherence and discontinuation for the generic medication cohort. $A H A$ antihyperglycemic agent, $C I$ confidence interval, $E R$ emergency room, $N E$ Northeast, $R R$ relative risk. The following covariates were controlled in the model at baseline: demographics (age, gender, race, and geographic regions), clinical factors

medications is significantly higher than for their generic counterparts ( $\$ 41$ vs. $\$ 6$, respectively). Consequently, a rise in copayment for branded medication may be more financially (comorbidities, Charlson Comorbidity Index, diabetesrelated complications), and economic factors (healthcare resource utilization, medication utilization, new AHA users, Part D benefit plan type, and out-of-pocket Part D costs)

prohibitive for the patient, whereas a comparable percentage increase in copayment for the generic medication results in a lower total cost and thus has less impact on their medication 
usage because these patients are less price sensitive to increases in lower cost medications. An anomaly observed in this study was that the relative risk of discontinuation in the generic cohort was lower for copayment values greater than zero than that observed for a zero copayment that had a relative risk of 1 . Although relative risk of discontinuation in the generic cohort was statistically significant, most of the relative risk ratios were close to 1 , so it is difficult to make any conclusive assessment of this association. This may be attributable to the large sample size; however, further studies are needed to better understand the association between magnitude of change in copay and the discontinuation rate of generic medications.

Among the modeled covariates, age, sex, race/ethnicity, region, new AHA usage, and health resource utilization in the preindex period were statistically significant predictors of nonadherence. Black and Hispanic patients, beneficiaries newly prescribed the index AHA, and those with more frequent inpatient, ER, and/or physician visits were all at higher risk of being nonadherent or discontinuing medication.

As observed in the current study, higher copayments have been shown in other studies to be associated with decreased adherence in adult patients with diabetes (included data from Medicaid claims, administrative claims databases, and employer-sponsored insurance databases) as well as increased overall healthcare costs due to the potential for poorer outcomes and additional medical procedures, medications, hospitalization, and healthcare resource use in these patients who are at risk for diabetesassociated complications including cardiovascular events [17-21]. A recent systematic review and meta-analysis, wherein all eligible studies were retrospective cohort studies, assessed the association among medication adherence and cardiovascular disease, all-cause mortality, and all-cause hospitalization in patients with T2D [18]. The analysis found that the relative risk of good adherence (defined as $\geq 80 \%$ ) versus poor adherence was 0.72 (95\% CI $0.62-0.82$; $P<0.001)$ for all-cause mortality and $0.90(95 \%$ CI $0.87-0.94 ; P<0.001)$ for hospitalization. An analysis of Medicaid claims data in the North
Carolina Medicaid program showed that a policy change to increase copayments for brandname medications not only decreased medication adherence but also resulted in a net increase in Medicaid expenditures, including individuals taking AHAs [17]. In addition, a retrospective, cross-sectional study conducted in adult patients with diabetes taking an oral AHA with employer-sponsored insurance (Thomson Reuters MarketScan ${ }^{\circledR}$ Database; 2002-2006) [19] showed that medication adherence resulted in lower rates of diabetesrelated complications (amputation/ulcer, acute myocardial infarction, retinopathy, and neuropathy), reduced ER visits, and fewer inpatient admissions. A retrospective cohort study in Medicaid beneficiaries with T2D (North Carolina Medicaid database; 1 July 2000-30 June 2003) reported a statistically significant $(P<0.01)$ reduction in diabetes-related costs of $4 \%$ and a $2 \%$ reduction in total healthcare costs for each $10 \%$ increase in adherence $(P<0.001)$ [20]. Moreover, Encinosa et al. found that increasing medication adherence in patients with T2D from $50 \%$ to $100 \%$ reduced hospitalization by $23.3 \%$ and ER visits by $46.2 \%$ [21]. Increased adherence resulted in an increased drug cost of $\$ 766$ per patient; however, this increase was offset by a cost savings of $\$ 866$ per patient due to a reduction in hospitalization and ER visits [21].

Higher copayments alone may not always result in decreased adherence because the primary determinant is more likely to be the final overall cost to the patient. This observation is supported by a recent study by Stuart et al. evaluating the effect of Medicare Part D cost sharing on low-income subsidy recipients with diabetes [22]. This study showed that higher copayments for branded or generic oral AHAs or statins had little association with drug utilization by low-income subsidy recipients. One potential explanation for this finding was that copayments for branded as well as generic medications are significantly lower for lowincome subsidy recipients than other populations covered under Medicare, showing low sensitivity to costs.

The negative impact of higher copayments on medication adherence has been previously 
reported in studies in other disease states. A retrospective cohort study of patients with chronic heart failure (CHF) who were receiving commercial or Medicare supplemental benefits for prescription antihypertensive medications showed that higher medication copayments were associated with poorer adherence and increased the predicted risk of hospitalization for patients with CHF [23]. Depending on the class of antihypertensive agent, a $\$ 10$ increase in copayment for Medicare beneficiaries was associated with a decrease in the measure of adherence by $1.8-2.6 \%$ and a predicted $6.1-8.7 \%$ increase in the risk of hospitalization for $\mathrm{CHF}$, which increases healthcare costs because of increased utilization reimbursements. Gibson et al. used medical and pharmacy claims data among continuously enrolled statin users from the 2000-2003 Medstat MarketScan database to estimate the effects of copayment changes on statin adherence [24]. Higher copayments were associated with lower adherence, and a copayment increase going into effect all at once had a greater impact on adherence than the same copayment increase going into effect over time. Consequently, higher copayments were a financial barrier to statin adherence, and short-term savings in program costs might potentially be outweighed by increased cardiovascular events and additional clinical procedures resulting from statin nonadherence [24]. Similarly, higher copayments have been associated with decreases in adherence in Medicaid beneficiaries (0.16-4.6 percentage points) $[17,25,26]$, veteran beneficiaries (2.0-7.0 percentage points) $[25,27,28]$, and patients with commercial insurance (1.8-2.0 percentage points) [13, 23, 25, 29, 30].

Diabetes is a major concern in the elderly and is estimated to occur in one-third of this fragile population [31]. Elderly patients with diabetes have a high prevalence of diseaseassociated complications such as heart disease, peripheral vascular disease, and stroke, as well as microvascular comorbidities [32]. The presence of diabetes also increases the risk of depression, dementia, falls, and incontinence in this population [32]. As the proportion of elderly individuals continues to grow, successful management of diabetes must include maintaining high levels of adherence to reduce the incidence and severity of diabetes-associated complications and healthcare costs. A retrospective analysis using a large Medicare claims database (Truven Medicare Supplemental database; data from 1 July 2009 to 30 June 2014) evaluated the association between adherence and outcomes in patients aged $\geq 65$ years with T2D $(N=123,235)$ [33]. Study results showed that higher AHA adherence rates were associated with a substantial decreases in total medical costs that were driven by reduced acute care costs despite higher drug costs. Comparing most-adherent with least-adherent patients was estimated to result in a reduction in the probability of a hospitalization or an ER visit of approximately $19 \%$ and $18 \%$, respectively, as well as a $50 \%$ reduction in the number of hospitalizations or ER visits, and approximately only $33 \%$ of the hospital length of stay time.

Payers should consider these findings regarding the relationship between adherence and medication copayments when making formulary decisions. Increasing fiscal pressure to control federal health insurance spending in programs such as Medicaid and Medicare using short-term cost-saving measures such as copayment increases may have a detrimental effect on medication adherence. Payers, policy makers, and insurance plans would benefit from instituting measures to encourage and maintain good adherence in patients with $\mathrm{T} 2 \mathrm{D}$ as decreased healthcare costs and improvements in patient outcomes are beneficial to the entire healthcare system.

\section{LIMITATIONS}

This study has a number of potential limitations pertaining to the use of administrative claims data including coding errors, measurement limitations (accuracy of diagnostic, procedural, and prescription drug codes), and the unknown actual consumption of dispensed medications per pharmacy claims. Additionally, the majority of patients were on a generic medication $[n=131,594$ (82\% of total population) $]$ compared with a branded $[n=28,656(18 \%)]$ medication. Thus, although both of the sample 
populations were large, some of the stratification by copayment categories led to small sample size, which may have impacted the results. Furthermore, the overall health status of the generic cohort was better compared with the branded cohort, which could have introduced potential bias into the analysis. Another potential study limitation is that medication discontinuation was defined as a treatment gap of $>60$ days; although this is a commonly used criterion in the literature, it is an arbitrarily chosen time period. Consequently, this length of time may not be comparable to discontinuation rates reported in other studies and also does not account for patients who may have reinitiated treatment after the 60-day gap. Furthermore, the generalizability of this study data is limited because the study population was composed of continuously enrolled Medicare fee-for-service beneficiaries; thus, extrapolation to other populations may not be appropriate. In addition, the analyses are limited to information obtained in administrative claims data, so the data may be incomplete or limited. For example, information on diagnoses, laboratory measures, and prescriptions outside of this system may not be captured in the databases. Moreover, educational and social factors (including race/ethnicity, socioeconomics, employment/income) may potentially be mitigating factors in cost-related adherence assessments; however, such data are not collected in Medicare claims databases and thus these factors could not be assessed in this study. Similarly, the duration of diabetes for patients in the branded and generic cohorts could not be determined for comparison because these data were not collected in the database. Finally, this study only evaluated the impact of copayment for patients on monotherapy. Thus, future research is needed to understand the relationship between copayment and nonadherence and discontinuation for patients on multiple medications.

\section{CONCLUSIONS}

The association between copayment and adherence and discontinuation varied considerably for branded versus generic AHAs among Medicare beneficiaries with T2D. Increase in the copayment was associated with a decrease in adherence and a higher discontinuation rate for branded AHA medications, but this phenomenon was not observed for generic medications. These results should be considered by payers when making formulary decisions as short-term cost-saving measures for the payer, such as copayment increases, may have a detrimental effect on medication adherence and subsequent health outcomes for patients and potential long-term costs for the healthcare system. Instituting measures to encourage and maintain good adherence in patients with T2D is beneficial to the entire healthcare system.

\section{ACKNOWLEDGEMENTS}

Funding. The study and manuscript processing charges were funded by Merck \& Co., Inc., Kenilworth, NJ, USA. All authors had full access to all of the data in this study and take complete responsibility for the integrity of the data and accuracy of the data analysis.

Editorial Assistance. Editorial support for development of this manuscript was provided by Alan J. Klopp, PhD, and Lisa Callan, PhD, at C4 MedSolutions, LLC (Yardley, PA), a CHC Group company, and was funded by Merck \& Co., Inc., Kenilworth, NJ, USA.

Authorship. All named authors meet the International Committee of Medical Journal Editors (ICMJE) criteria for authorship for this article, take responsibility for the integrity of the work as a whole, and have given their approval for this version to be published. Coauthors from Merck \& Co., Inc., Kenilworth, NJ, USA, were involved in providing input on study design; analysis, and interpretation of data; writing of the report; and the decision to submit the report for publication.

Prior Presentation. American Diabetes Association's 77th Scientific Sessions, San Diego, CA; June 9-13, 2017. 
Disclosures. Manjiri Pawaskar is an employee of Merck \& Co., Inc., Kenilworth, NJ, USA. Yuexin Tang is an employee of Merck \& Co., Inc., Kenilworth, NJ, USA. Swapnil Rajpathak is an employee of Merck \& Co., Inc., Kenilworth, NJ, USA. Liou Xu, Gary Puckrein, and Bruce Stuart declare that they have no conflicts of interest.

Compliance with Ethics Guidelines. This article is based on administrative claims data and does not contain any studies with human participants performed by any of the authors.

Data Availability. The data set used for this study was generated from the Centers for Medicare and Medicaid Services (CMS) database and is available from CMS (https://www.cms. gov/) following execution of a data use agreement.

Open Access. This article is distributed under the terms of the Creative Commons Attribution-NonCommercial 4.0 International License (http://creativecommons.org/licenses/ by-nc/4.0/), which permits any noncommercial use, distribution, and reproduction in any medium, provided you give appropriate credit to the original author(s) and the source, provide a link to the Creative Commons license, and indicate if changes were made.

\section{REFERENCES}

1. Centers for Disease Control and Prevention. National Diabetes Statistics Report, 2017. Estimates of Diabetes and its Burden in the United States. [cited 2017 September 28]. https://www.cdc.gov/diabetes/pdfs/ data/statistics/national-diabetes-statistics-report.pdf.

2. Stratton IM, Adler AI, Neil HA, et al. Association of glycaemia with macrovascular and microvascular complications of type 2 diabetes (UKPDS 35): prospective observational study. BMJ. 2000;321:405-12.

3. Asche C, LaFleur J, Conner C. A review of diabetes treatment adherence and the association with clinical and economic outcomes. Clin Ther. 2011;33:74-109.

4. Rozenfeld Y, Hunt JS, Plauschinat C, Wong KS. Oral antidiabetic medication adherence and glycemic control in managed care. Am J Manag Care. $2008 ; 14: 71-5$.

5. Fitch K, Pyenson BS, Iwasaki K. Medical claim cost impact of improved diabetes control for Medicare and commercially insured patients with type 2 diabetes. J Manag Care Pharm. 2013;19(609-20): 20a-20d.

6. Ali MK, Bullard KM, Saaddine JB, Cowie CC, Imperatore G, Gregg EW. Achievement of goals in U.S. diabetes care, 1999-2010. N Engl J Med. 2013;368:1613-24.

7. National Committee for Quality Assurance. The state of health care quality report 2015. 2015 [cited 2017 September 28]. http://store.ncqa.org/index. $\mathrm{php} / \mathrm{catalog} /$ product/view/id/2341/s/2015-state-ofhealth-carequality-report/.

8. Edelman SV, Polonsky WH. Type 2 diabetes in the real world: the elusive nature of glycemic control. Diabetes Care. 2017;40:1425-32.

9. McEwan P, Foos V, Palmer JL, Lamotte M, Lloyd A, Grant D. Validation of the IMS CORE diabetes model. Value Health. 2014;17:714-24.

10. Baxter M, Hudson R, Mahon J, et al. Estimating the impact of better management of glycaemic control in adults with type 1 and type 2 diabetes on the number of clinical complications and the associated financial benefit. Diabet Med. 2016;33:1575-81.

11. Curtis SE, Boye KS, Lage MJ, Garcia-Perez LE. Medication adherence and improved outcomes among patients with type 2 diabetes. Am J Manag Care. 2017;23:e208-14.

12. Gu Q, Zeng F, Patel BV, Tripoli LC. Part D coverage gap and adherence to diabetes medications. Am J Manag Care. 2010;16:911-8.

13. Colombi AM, Yu-Isenberg K, Priest J. The effects of health plan copayments on adherence to oral diabetes medication and health resource utilization. J Occup Environ Med. 2008;50:535-41.

14. Barron J, Wahl P, Fisher M, Plauschinat C. Effect of prescription copayments on adherence and treatment failure with oral antidiabetic medications. Pharm Therapeut. 2008;33:532-53.

15. Peterson AM, Nau DP, Cramer JA, Benner J, Gwadry-Sridhar F, Nichol M. A checklist for medication compliance and persistence studies using retrospective databases. Value Health. 2007;10:3-12.

16. Charlson ME, Pompei P, Ales KL, MacKenzie CR. A new method of classifying prognostic comorbidity in longitudinal studies: development and validation. J Chronic Dis. 1987;40:373-83. 
17. Domino ME, Martin BC, Wiley-Exley E, et al. Increasing time costs and copayments for prescription drugs: an analysis of policy changes in a complex environment. Health Serv Res. 2011;46:900-19.

18. Khunti K, Seidu S, Kunutsor S, Davies M. Association between adherence to pharmacotherapy and outcomes in type 2 diabetes: a meta-analysis. Diabetes Care. 2017;40:1588-96.

19. Gibson TB, Song X, Alemayehu B, et al. Cost sharing, adherence, and health outcomes in patients with diabetes. Am J Manag Care. 2010;16:589-600.

20. Shenolikar RA, Balkrishnan R, Camacho FT, Whitmire JT, Anderson RT. Comparison of medication adherence and associated health care costs after introduction of pioglitazone treatment in African Americans versus all other races in patients with type 2 diabetes mellitus: a retrospective data analysis. Clin Ther. 2006;28:1199-207.

21. Encinosa WE, Bernard D, Dor A. Does prescription drug adherence reduce hospitalizations and costs? The case of diabetes. Adv Health Econ Health Serv Res. 2010;22:151-73.

22. Stuart B, Hendrick FB, Xu J, Dougherty JS. How lowincome subsidy recipients respond to Medicare Part D cost sharing. Health Serv Res. 2017;52:1185-206.

23. Cole JA, Norman H, Weatherby LB, Walker AM. Drug copayment and adherence in chronic heart failure: effect on cost and outcomes. Pharmacotherapy. 2006;26:1157-64.

24. Gibson TB, Mark TL, McGuigan KA, Axelsen K, Wang S. The effects of prescription drug copayments on statin adherence. Am J Manag Care. 2006;12:509-17.

25. Amin K, Farley JF, Maciejewski ML, Domino ME. Effect of Medicaid policy changes on medication adherence: differences by baseline adherence. J Manag Care Spec Pharm. 2017;23:337-45.

26. Lieberman DA, Polinski JM, Choudhry NK, Avorn J, Fischer MA. Unintended consequences of a Medicaid prescription copayment policy. Med Care. 2014;52:422-7.

27. Doshi JA, Zhu J, Lee BY, Kimmel SE, Volpp KG. Impact of a prescription copayment increase on lipid-lowering medication adherence in veterans. Circulation. 2009;119:390-7.

28. Maciejewski ML, Bryson CL, Perkins $\mathrm{M}$, et al. Increasing copayments and adherence to diabetes, hypertension, and hyperlipidemic medications. Am J Manag Care. 2010;16:e20-34.

29. Chernew ME, Shah MR, Wegh A, et al. Impact of decreasing copayments on medication adherence within a disease management environment. Health Aff (Millwood). 2008;27:103-12.

30. Gibson TB, Mark TL, Axelsen K, Baser O, Rublee DA, McGuigan KA. Impact of statin copayments on adherence and medical care utilization and expenditures. Am J Manag Care. 2006;12:SP11-SP19.

31. Cowie CC, Rust KF, Ford ES, et al. Full accounting of diabetes and pre-diabetes in the U.S. population in 1988-1994 and 2005-2006. Diabetes Care. 2009;32:287-94.

32. Corriere M, Rooparinesingh N, Kalyani RR. Epidemiology of diabetes and diabetes complications in the elderly: an emerging public health burden. Curr Diab Rep. 2013;13:805-13.

33. Boye KS, Curtis SE, Lage MJ, Garcia-Perez LE. Associations between adherence and outcomes among older, type 2 diabetes patients: evidence from a Medicare Supplemental database. Patient Prefer Adherence. 2016;10:1573-81. 